\title{
Study on Building and Modeling of Virtual Data of Xiaosha River Artificial Wetland
}

\author{
Qun Miao, Lei Bian, Xipeng Wang, Chang Xu
}

School of Environment and Municipal Engineering, Qingdao Technological University, Qingdao, China.

Email: lgdmq@163.com, leileibian@163.com

Received 2013

\begin{abstract}
From the viewpoint of systems science, this article takes Xiaosha River artificial wetland under planning and construction as object of study based on the systems theory and takes the accomplished and running project of Xinxuehe artificial wetland as reference. The virtual data of quantity and quality of inflow and the quality of outflow of Xiaosha River artificial wetland are built up according to the running experience, forecasting model and theoretical method of the reference project as well as the comparison analysis of the similarity and difference of the two example projects. The virtual data are used to study the building of forecasting model of BP neural network of Xiaosha River artificial wetland.
\end{abstract}

Keywords: Xiaosha River Artificial Wetland; Virtual Data; BP Neural Network

\section{Introduction}

To build the model of an artificial wetland, we need abundant measured data. But as Xiaosha river artificial wetland is under planning and construction, it's impossible to obtain the experimental data. Therefore we may use virtual experimental data to satisfy the need of modeling of Xiaosha river artificial wetland. The building of the virtual data shall be based on the similarity and difference between the object of study (Xiaosha river artificial wetland) and the reference project (Xinxuehe artificial wetland). The virtual data of Xiaosha river artificial wetland shall be built up according to the running experience and forecasting model of the reference project and used for building the forecasting model of BP neural network of Xiaosha river artificial wetland.

\section{Building of the Inflow Quantity Virtual Data of Xiaosha River Artificial Wetland}

According to the monitoring documents of the reference project Xinxuehe artificial wetland, the inflow quantity of the running wetland shows an important variation with change of season and certain regularity and the real inflow rate floats up and down from the designed inflow rate. Therefore, the virtual data of the inflow quantity of Xiaosha river artificial wetland is built up virtually according to the ratio of real inflow quantity to designed inflow quantity and by comparing the data of the same period (between 2008 and 2009). The virtual values of inflow quantity are given in Table $\mathbf{1}$.

\section{Building of the Outflow Quality Virtual Data of Xiaosha River Artificial Wetland}

\subsection{Modeling of Outflow Quality of the Reference Project}

It is assumed that Xiaosha river artificial wetland is completely the same as the reference project. The established forecasting model of the water quality of BP neural network of the reference project Xinxuehe artificial wetland is used to forecast the outflow quality data of Xiaosha river artificial wetland.

Table 1. Table type styles (Table caption is indispensable).

\begin{tabular}{ccccc}
\hline \multirow{4}{*}{$\begin{array}{c}\text { Monitoring } \\
\text { month }\end{array}$} & $\begin{array}{c}\text { Virtual } \\
\text { Inflow } \\
\text { quantity } \\
\left(\mathrm{m}^{3} / \mathrm{d}\right)\end{array}$ & $\begin{array}{c}\text { Ratio of virtual } \\
\text { inflow ratel } \\
\text { designed } \\
\text { inflow rate }\end{array}$ & $\begin{array}{c}\text { Virtual in- } \\
\text { flow } \\
\text { quantity } \\
\left(\mathrm{m}^{3} / \mathrm{d}\right)\end{array}$ & $\begin{array}{c}\text { Ratio of virtual } \\
\text { inflow ratel } \\
\text { designed } \\
\text { inflow rate }\end{array}$ \\
\hline 2 & 68200 & 2.20 & 76570 & 2.47 \\
3 & 59780 & 0.98 & 57340 & 0.94 \\
4 & 48800 & 0.80 & 44530 & 0.73 \\
5 & 55510 & 0.91 & 50630 & 0.83 \\
6 & 184830 & 3.03 & 123220 & 2.02 \\
7 & 159210 & 2.61 & 154330 & 2.53 \\
8 & 169580 & 2.78 & 233630 & 3.83 \\
9 & 117120 & 1.92 & 189100 & 3.10 \\
10 & 62830 & 1.03 & 175680 & 2.88 \\
11 & 62310 & 2.01 & 107570 & 3.47 \\
12 & 55180 & 1.78 & 71300 & 2.30 \\
\hline
\end{tabular}

Note.: In this area, January is the freezing period and there is no inflow into the wetland in this month. 
According to the virtual data building method, the building of the outflow quality data of Xiaosha river artificial wetland is based on the forecasting model of the reference project Xinxuehe artificial wetland, i.e. the inflow data of Xiaosha river can be taken as input to forecast the outflow quality of Xiaosha river artificial wetland when the two projects are completely the same. Therefore, it's necessary to build up a forecasting model of BP neural network of the reference project with inflow quantity and quality as input and outflow quality as output. The forecasting model is built up for three seasons of February $\sim$ May, June $\sim$ September and October $\sim$ December.

This model is in a structure of a three-layer neural network[1], in which there're 3 input layer neural elements and 2 output layer neural elements, the transfer function of the hidden layer neural element is logsig, the transfer function of the output layer neural element is purelin, the maximum number of study cycles of the network is 10000 , the target error is 0.001 , the study speed is 0.1 . For the three seasons, the number of nodes is 9,11 and 11 respectively and the training function of the network is trainrp, trainscg and trainrp. The relative error of the sample test is within the acceptable range after testing. The relative error of the forecasting of water quantity, CODCr and $\mathrm{NH}_{3}-\mathrm{N}$ of the neural network model of February May is $4.46 \%$ and $7.34 \%$; the rela- tive error of the forecasting of the model of June $\sim$ September is $6.00 \%$ and $7.21 \%$; the relative error of the forecasting of the model of October December is $6.94 \%$ and $7.97 \%$ respectively.

The error of the forecasting model in each season of the reference project is from $-11.38 \%$ to $12.43 \%$. According to the criterion rule of the forecasting model [2][3], the training result is acceptable when the relative error is below $30 \% \sim 40 \%$ during the analog forecasting of BP neural network. Therefore, the network training is successful and its performance can meet the requirement of real application and can be used to build the virtual data of Xiaosha river artificial wetland.

\subsection{Building of Outflow Quality Virtual Data of Xiaosha River Artificial Wetland}

IXiaosha river artificial wetland and the reference project are similar and different at the same time. These differences decide that the outflow quality of the wetland is different when the inflow quantity and quality are the same for the two projects. Therefore, the outflow quality virtual data of Xiaosha river artificial wetland need to be adjusted according to the difference between the two projects. The adjusted data are the outflow quality virtual data of Xiaosha river artificial wetland.

The analysis shows that the difference consists in the optimization of the design of Xiaosha river artificial wetland, mainly including the enlargement of wetland size, the regularity of geometry of wetland and the reinforcement of wetland function zone. The determination of the favorable impact of the three optimizing factors on the eliminating rate of pollutant of the wetland in each season needs to be obtained from the analysis of abundant experimental data. But Nansihu basin wetland ecological recovery project is now under demonstration study phase, so it's only possible to make the above qualitative analysis on the favorable impact of the three optimizing factors in each season, but not possible to make quantitative determination by using mathematics method. Therefore, it's necessary to transform the qualitative analysis into quantitative analysis to realize the building of outflow quality virtual data of Xiaosha river artificial wetland. This article transforms the qualitative analysis into quantitative analysis by using expert rating method.

To make the adjusting coefficient as accurate as possible, during the study, we invited some experts who have many years of study experience to rate for the artificial wetland. Before rating, we provided them with the documents related to the construction and running of the two example projects and the experts rated for the two indices $\mathrm{COD}_{\mathrm{Cr}}$ and $\mathrm{NH}_{3}-\mathrm{N}$ according to their own study experience and result [4-5].

The statistics result of the experts' ratings shows that the adjusting coefficient of $\mathrm{COD}_{\mathrm{Cr}}$ of the outflow quality data of Xiaosha river artificial wetland from February to May is 0.72 ; the adjusting coefficient of $\mathrm{COD}_{\mathrm{Cr}}$ from June to September is 0.42; the adjusting coefficient of $\mathrm{COD}_{\mathrm{Cr}}$ from October to December is 0.47 . The adjusting coefficient of $\mathrm{NH}_{3}-\mathrm{N}$ from February to May is 0.82 ; the adjusting coefficient of $\mathrm{NH}_{3}-\mathrm{N}$ from June to September is 0.61 ; the adjusting coefficient of $\mathrm{NH}_{3}-\mathrm{N}$ from October to December is 0.72 . Therefore, the outflow quality virtual data of Xiaosha river artificial wetland in 2008 and 2009 are given in Table 2.

\section{Building of Neural Network Forecasting Model of Xiaosha River Artificial Wetland}

The BP neural network forecasting model apt for Xiao sha river artificial wetland is built up for three seasons of February $\sim$ May, June $\sim$ September and October $\sim$ December based on the virtual data of Xiaosha river artificial wetland and using matlab software.

The forecasting model of Xiaosha river artificial wetland is a three-layer neural network, in which there're 2 input layer neural elements and 3 output layer neural elements. After repetitive trials: the number of the hidden layer neural elements for model February May is 9; the number of neural elements of June $\sim$ September is 12; the number of neural elements of October December is 13 . The parameters of the forecasting model of Xiaosha river artificial wetland are determined as: the maximum number 
Table 2. Outflow quality virtual data of Xiaosha river artificial wetland.

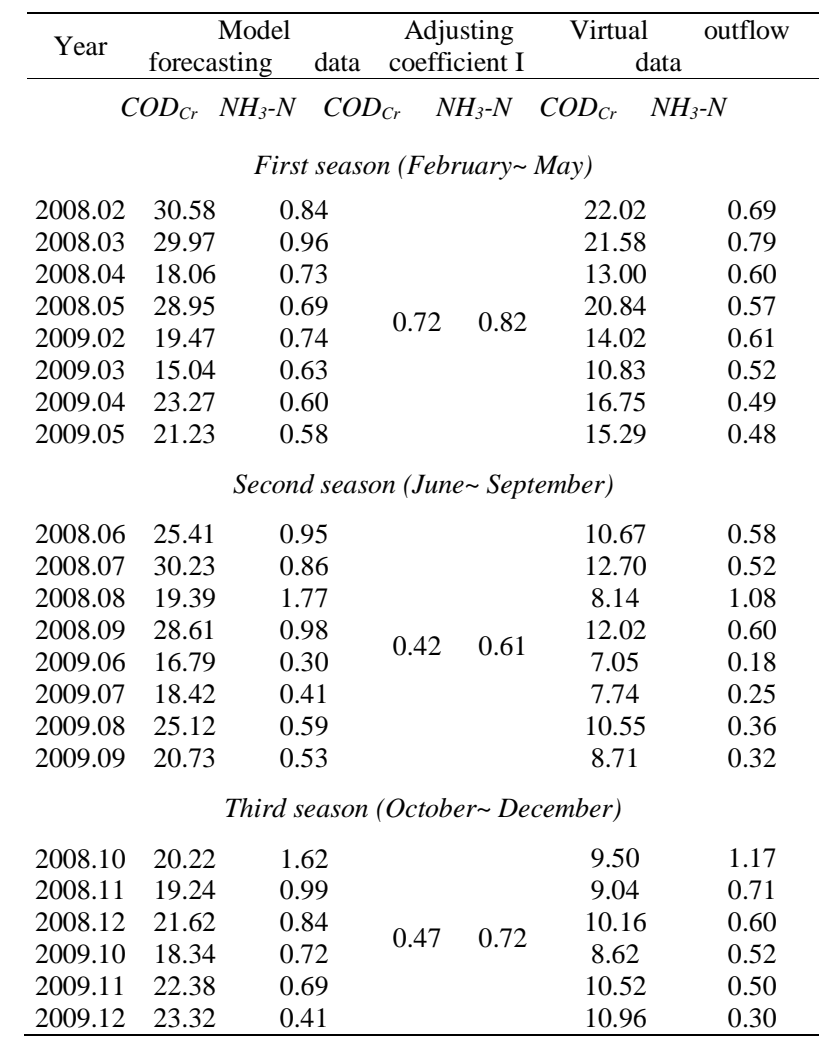

of study cycles is 10000; target error is 0.001 ; study speed is 0.1 ; transfer function of output layer neural elements is purelin; transfer function of hidden layer neural elements is logsig. The training functions for neural network of each season are: trainrp for model of February May, trainrp for model of June September, traingda for model of October $\sim$ December.

The virtual data test samples are used for testing and verification of the neural network forecasting model of each season. The testing result shows that for Xiaosha river artificial wetland, the relative error of water quantity of model February May is 9.35\%; the relative errors of $\mathrm{COD}_{\mathrm{Cr}}$ and $\mathrm{NH}_{3}-\mathrm{N}$ are $12.96 \%$ and $9.55 \%$ respectively; the relative error of water quantity of model June $\sim$ September is $19.25 \%$; the relative errors of $\mathrm{COD}_{\mathrm{Cr}}$ and $\mathrm{NH}_{3}-\mathrm{N}$ are $5.45 \%$ and $8.38 \%$ respectively; the relative error of water quantity of model October $\sim$ December is $12.10 \%$; the relative errors of $\mathrm{COD}_{\mathrm{Cr}}$ and $\mathrm{NH}_{3}-\mathrm{N}$ are $11.90 \%$ and $19.11 \%$ respectively, so the model training meets the requirement. But after running of Xiaosha river artificial wetland, it's still necessary to get real monitored data to test and correct the model.

\section{Conclusions}

1) We take the inflow quantity and quality virtual data of Xiaosha river artificial wetland as input and use the outflow quality forecasting model of the reference project to forecast and obtain the outflow quality data of Xiaosha river artificial wetland when the two example projects are completely the same.

2) According to the difference with the reference project, we analyze the impact of the three optimizing design factors including enlargement of wetland size, regularity of geometry of wetland and reinforcement of wetland function zone on the elimination rate of pollutant and use expert rating method to make quantitative analysis on the impact and determine the adjusting coefficient in order to realize the building of the outflow quality virtual data of Xiaosha river artificial wetland.

3) We use the virtual data test samples to test and verify the neural network forecasting model of each season. The test result shows that the model training meets the requirement. But after running of Xiaosha river artificial wetland, it's still necessary to get real monitored data to test and correct the model.

\section{Acknowledgements}

This study is sponsored by the project of "Nansihu deteriorated wetland ecological recovery and water quality improveement technology and demonstration" (No. 2009ZX07210-009) which is an important specific project of national water body pollution control.

\section{REFERENCES}

[1] Nan Liang, "Neural Network And the Fuzzy Control," Beijing, Tsinghua University Press,2000.

[2] Nan Liang, "Water Quality Forecast and Realization By MATLAB Based On Artificial Neural Network,” Chang'an University,2007.

[3] Xiaoping Wang, Jiyang Sun, Xin Jin, "Forecast of Water Quality Indices of Qiantang River Based on BP Neural Network," Zhejiang University Journal (Engineering), 2007.41 (2) , pp. 361-364.

[4] HuangXinRong, "Qian xuesen, Complexity THoughts study,” 2004.10,12 (4).

[5] MiaoDongSheng, "Qian xuesen Complexity Research Review,” Journal of xi 'an jiaotong university(social science edition), 2004.12,24 (4). 